\title{
A compact equipment for the sulfur content estimation in heavy oil using neutrons and gamma-rays
}

\author{
Pino F. ${ }^{1,2}$, Stevanato L. ${ }^{2}$, Fabris D. ${ }^{3}$, Barros H. ${ }^{1}$, Vidal A. ${ }^{1}$, Nebbia G. ${ }^{3}$, Lunardon M. ${ }^{2}$, \\ Moretto S. ${ }^{2}$, Sajo-Bohus L. ${ }^{1,2}$ \\ ${ }^{1}$ Universidad Simon Bolivar, Ap.do 89000, 1080A Caracas, Venezuela \\ ${ }^{2}$ Dipartimento di Fisica ed Astronomia dell'Università di Padova, I-35131 Padova, Italy \\ ${ }^{3}$ INFN Sezione di Padova, Via Marzolo 8, I-35131 Padova, Italy
}

A compact and modern equipment for fast neutron and $\gamma$-ray transmission (FNGT) technique has been developed. The FNGT is used to perform non-destructive analysis of samples and it makes use of R-ratio that is the ratio of absorption coefficients of fast neutrons and gamma-rays [1].

The FNGT technique has a very good resolving power to determine the average atomic number of compounds with low atomic number $(\mathrm{Z}<13)$. Crude oil lays in this range of values, therefore we suggest to employ the mentioned technique for monitoring sulfur content in oil-business. The on-line analysis of fluid provides knowledge of sulfur content that has positive effects on the refinery plant. Nowadays, the used technique requires 7-10 days of delay in getting the information due the offline chemical or nuclear analysis.

The compact system makes use of a Cf-252 source, a single liquid scintillator detector EJ-301 with neutron/gamma discrimination capabilities and a modern nuclear electronics based on fast digitizers.

The use of an optimized Pulse Shape Discrimination algorithm provides a good separation of gammas from neutrons; the method is an alternative to that obtainable by TOF-technique, widely used in the past, that requires almost 1 meter of time of flight and another detector for triggering the fission events. The compactness of this assembly ensures a higher counting efficiency, offering the advantage of a shorter acquisition time to get sufficiently high statistic.

The fast neutron and gamma transmission technique was tested in laboratory for online sulfur concentration estimation in a sample of Venezuelan heavy oil. The range of sulfur concentration investigated is between $0.1 \%-6.5 \%$ in weight. The experimental setup performance and limitations are compared with those predicted by a Monte Carlo model built in GEANT4 v10.01. In particular, we studied with Monte Carlo simulations the radiation buildup effect on the R-values and on the overall sensitivity of the technique. In fact, in the used experimental set-up, the Cf- 252 source was not collimated in a "narrow beam", leaving to the detector the possibility to respond either to neutrons and $\gamma$-rays directly from the source or from scattered radiation.

The results show that this technique for on-line characterization of the crude oil copes well with the industrial requirements in terms of precision and response time, representing then an interesting alternative to the standard procedures.

\section{The Sulfur-in-Oil Experiment}

The experimental set-up was a compact system made of a Cf-252 neutron source, a single liquid scintillator detector with good response for both fast neutrons and $\gamma$-rays and a modern nuclear electronic setup based on fast digitizers (for digital processing of the signals). A Monte Carlo model based on the GEANT4 v10.01 code was built in order 
to optimize and evaluate the response and the efficiency of the system.

The sample and radioisotope source holder are positioned at $30 \mathrm{~cm}$ from the front-face of the scintillator detector. The detector is of a cylindrical EJ-301 liquid scintillator cell of $5 \mathrm{~cm}$ in diameter and $5 \mathrm{~cm}$ thickness, coupled to an H1949-51 HAMAMATSU linearly focused 12 dynodes photomultiplier (PMT) through an EJ-560 silicone optical interface. The operating voltage was set at $-1500 \mathrm{~V}$. The front-end electronics used in this work was composed of the following CAEN VME modules: a V6533 Programmable HV Power Supply (6 Ch., 4 kV, 3 mA, 9 W), a V1730 Digitizer (14 bits, $500 \mathrm{MS} / \mathrm{s}$ ), and a Bridge USB V1718 connected to a PC with a data acquisition software. Inside the digitizer, Digital Pulse Processing (DPP) algorithms are implemented using an FPGA, providing pulse online analysis for each event having: (a) a timestamp, (b) a complete integration of the signal, (c) a partial integration of the signal used for Pulse Shape Discrimination analysis and (d) the possibility of storing a selected part of the digitized signal. The optimization of the DPP parameters was obtained as described in [2]. A $10^{5}$ neutrons/s Cf- 252 source was used.

Mono-elemental samples (with thickness from 1 to $3 \mathrm{~cm}$ ) were used to calibrate the system as reported in [1]. A second group of compounds was used to simulate the composition of the crude oil with different sulfur contents (from 0 to $6 \mathrm{wt} \%$ ). The sample is made of a plastic cilindric container filled by common engine oil (with a negligible surfur content), and a sulfur disk of known mass attached on the top. An appropriate Monte Carlo simulation was carried out to demonstrate the negligible effect in using two compound's layer instead of a homogenously mixed sample.

\section{Results and discussion}

The data analysis was performed using ROOT software. Neutron/gamma discrimination was performed offline by setting a proper discrimination line in the PSD vs. Integratedcharge plot (see ref. [2] for the complete definition of the parameters and the neutron/gamma discrimination procedure). The separation line defines a neutron region with gamma contamination lower than $0.2 \%$ at all energies, calculated by measuring a pure ${ }^{22} \mathrm{Na}$ gamma source (see [2,3] for more details)

Each measurement performed in this work was obtained in a time interval of 15 minutes (time of flight technique requires around $6 \mathrm{~h}$ of acquisition time to get equivalent statistics).

Material recognition can be obtained from R-ratio $[1,4,5,6]$ based on absorption coefficients for fast neutrons $\mu_{\mathrm{n}}$, and $\gamma$-rays $\mu_{\gamma}$, or the logarithmic ratio of the measured transmission factors for neutrons $\left(\mathrm{I}_{\mathrm{n}} / \mathrm{I}_{\mathrm{n}, 0}\right)$ and gamma rays $\left(\mathrm{I}_{\gamma} / \mathrm{I}_{\gamma, 0}\right)$, respectively. Therefore the following relationship holds:

$$
\mathrm{R}=\mu_{\mathrm{n}} / \mu_{\gamma}=\ln \left(\mathrm{I}_{\mathrm{n}} / \mathrm{I}_{\mathrm{n}, 0}\right) / \ln \left(\mathrm{I}_{\gamma} / \mathrm{I}_{\gamma, 0}\right)
$$

where: $I_{n}$ and $I_{\gamma}\left(I_{n, 0}\right.$ and $\left.I_{\gamma, 0}\right)$ are measured yields for neutrons and $\gamma$-rays with (without) the sample. The most important outcome is that the R-ratio characterizes a selected sample independently of its thickness or geometrical shape.

In the $R$ value calculation the same threshold on light output was used both for laboratory measurements and Monte Carlo simulations. The value was set at 50 keVee. The uncertainties associated to the experimental R-values in this work are $2.2 \%$. This value can be calculated by error propagation, but was also verified experimentally. The mono-elemental and mixtures samples $\mathrm{R}$ values are obtained as a function of their effective atomic number and it is shown in Fig. 1, left panel. 

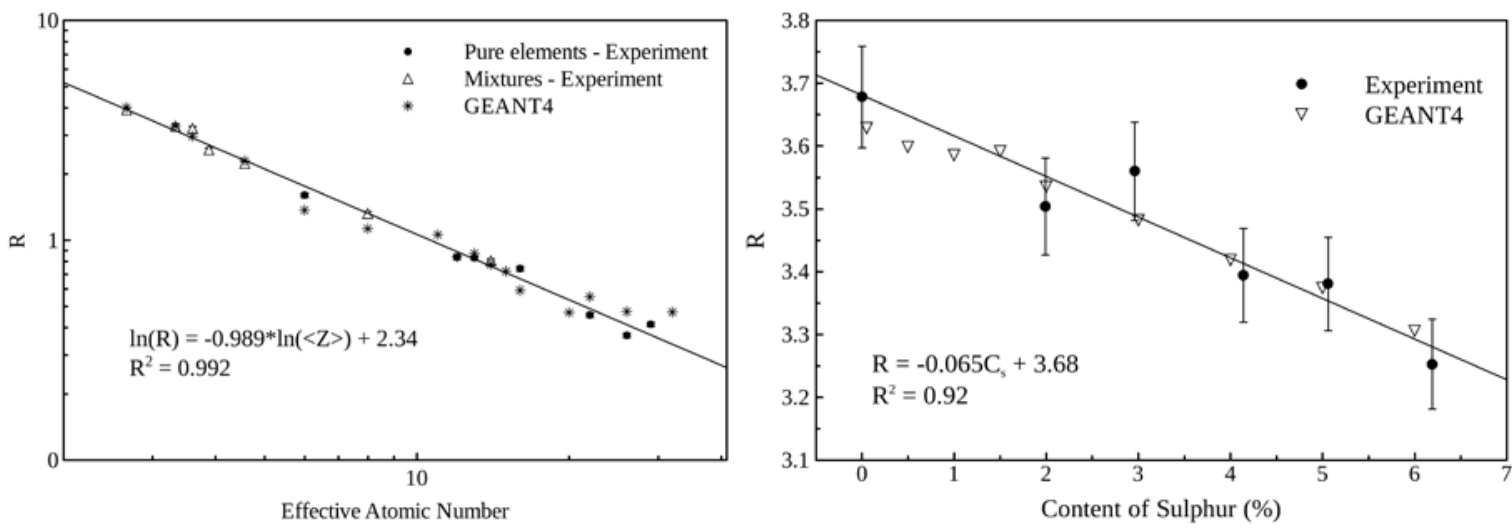

Fig. 1. R-ratio experimental results - see text fo details.

In Fig. 1, right panel, the experimental and Monte Carlo simulation results of the Rvalues associated to the crude oil samples with different sulfur concentrations are shown. The experimental R-values are well represented by a compound with an effective atomic number around $Z_{\text {eff }} \sim 3$, which is very close to the $Z_{\text {eff }}$ of crude oil (calculated according to its well-known major composition). Above $2 \%$ of sulfur content, a linear trend of the data is observed, with very good agreement with the simulation results.

Monte Carlo results indicate that the determination of the sulfur content for concentrations lower than $1.5 \%$ suffers high uncertainty, however, it is more stable and predictable the sulfur concentration in the region corresponding to heavy-sour crude oil, where the technique is intended to be used.

\section{Conclusions}

These results indicate the possibility to develop a compact and modern equipment for quality control of heavy-sour oil (sulfur content higher than 2\%) with the aim to provide a new technical tool to Venezuelan oil refineries. This non-destructive analysis to determine sulfur content has been demonstrated experimentally and proven by Monte Carlo simulation. The experimental results described in this paper show that this method is fully adequate in the range of average R-value corresponding to sulfur concentration of interest to the oil industrial refineries. This technique showed a lack of precision in the region of sulfur concentration below about $1.5 \%$, nevertheless a useful precision could be obtained increasing the source intensity within reasonable bounds (and keeping the short time intervals for the analysis).

\section{References}

[1] Viesti G, et al. (2008). Nucl. Instrum. Meth. A. DOI 10.1016/j.nima.2008.05.024

[2] Stevanato L, Cester D, Nebbia G, Viesti G, (2012). Nucl. Instrum. Meth. A. DOI 10.1016/j.nima.2012.06.047

[3] Stevanato L, et al. (2011). Appl. Radiat. Isotopes. DOI 10.1016/j.apradiso.2010.10.022

[4] Stevanato L, et al. (2013). Appl. Radiat. Isotopes. DOI 10.1016/j.apradiso.2012.11.018

[5] R.J. Rasmussen, et al. (1997). Nucl. Instr. and Meth. B. DOI 10.1016/S0168-583X(97)00120-1

[6] Cywicka-Jakiel T (2003). Applied Energy. DOI 10.1016/S0306-2619(02)00190-3 